\title{
Flux-driven Josephson parametric amplifier for sub-GHz frequencies fabricated with side-wall passivated spacer junction technology
}

\author{
Slawomir Simbierowicz ${ }^{1}$, Visa Vesterinen ${ }^{1,2}$, Leif \\ Grönberg $^{1}$, Janne Lehtinen ${ }^{1}$, Mika Prunnila ${ }^{1}$ and Juha \\ Hassel $^{1}$ \\ ${ }^{1}$ VTT Technical Research Centre of Finland Ltd \& QTF Centre of Excellence, \\ P.O.Box 1000, FI-02044 VTT, FINLAND \\ ${ }^{2}$ QCD Labs, COMP Centre of Excellence, Department of Applied Physics, \\ Aalto University, P.O. Box 13500, 00076 Aalto, Finland \\ E-mail: slawomir.simbierowicz@vtt.fi
}

May 2018

\begin{abstract}
We present experimental results on a Josephson parametric amplifier tailored for readout of ultra-sensitive thermal microwave detectors. In particular, we discuss the impact of fabrication details on the performance. We show that the small volume of deposited dielectric materials enabled by the side-wall passivated spacer niobium junction technology leads to robust operation across a wide range of operating temperatures up to $1.5 \mathrm{~K}$. The flux-pumped amplifier has gain in excess of $20 \mathrm{~dB}$ in three-wave mixing and its center frequency is tunable between $540 \mathrm{MHz}$ and $640 \mathrm{MHz}$. At $600 \mathrm{MHz}$, the amplifier adds $105 \mathrm{mK} \pm 9 \mathrm{mK}$ of noise, as determined with the hot/cold source method. Phase-sensitive amplification is demonstrated with the device.
\end{abstract}

Keywords: Josephson junction, parametric amplifier, SQUID array 


\section{Introduction}

In recent years, high-fidelity detection of radiofrequency (rf) and microwave signals that can consist of only a few photons has spun a lot of interest in the development of low-noise amplifiers. Such weak signals are encountered for instance in the search for dark-matter particles [1-3], fast readout of quantum bits (qubits) [4-7], and characterization of low-loss resonators [8] or nano-mechanical systems [9]. A promising branch of superconducting amplifiers, with near quantum-limited noise performance, exploits parametric pumping of the non-linear inductances exhibited by Josephson junctions [10-16] or those intrinsic to superconductors [17]. The Josephson parametric amplifier (JPA) has also proven to be capable of generating and using squeezed electromagnetic states [18-20] to go below the standard quantum limit of noise added by an amplifier [21].

Although the most common applications for the JPA are in the frequency band of $4-8 \mathrm{GHz}$, we recently reported on a JPA for $600 \mathrm{MHz}$ [22] to be used in conjunction with a nano-calorimeter $[23,24]$ or a -bolometer $[25,26]$ with a matching readout frequency. Our main motivation to develop the JPA is to allow the calorimeter to reach the accuracy of a single microwave photon and set a new record for the noiseequivalent power going below $10^{-19} \mathrm{~W} / \sqrt{\mathrm{Hz}}[27,28]$ in the bolometric mode. More recently, rf reflectometry of charge qubits has also emerged as a possible use case for the sub-GHz JPA [29]. Aiming to serve such applications, the realized JPA utilized the nonlinearity of niobium-based superconducting quantum interference devices (SQUIDs) in a lumped-element rf resonator. The amplifier of Ref. 22 was narrowband, but the center frequency of the gain was designed to be tunable with an external magnetic flux. However, the device suffered from multiple issues that prohibited its immediate use in calorimetry.

The first prominent issue discovered in Ref. 22 was an ill-behaved, hysteretic response of the resonance frequency to the applied magnetic flux. Its origin was attributed to flux trapping in the device geometry. The second issue was a high sensitivity to changes in the operating temperature, requiring stabilization of the JPA with closed-loop temperature control. We believe that the temperature sensitivity stemmed from twolevel systems (TLSs) in a deposited dielectric layer that had a large participation ratio to the JPA resonance.
This layer was made of silicon dioxide that is notorious for its high TLS density which significantly affects material properties at millikelvin temperatures [30, 31].

Here, we seek to improve on the shortcomings of Ref. 22 while keeping the design conceptually similar. We present several important modifications to the JPA, the first of which is the fabrication of the Josephson junctions with the so-called side-wall passivated spacer (SWAPS) process that we introduced recently [32]. This enables us to largely avoid using plasma-enhanced chemical vapour deposited (PECVD) silicon dioxide which is a necessity in our standard niobium tunnel junction processes [33]. Measures to control the flux trapping are implemented as well. The new JPA also employs three-wave mixing with an rf flux pump at twice the signal frequency [34] as opposed to the fourwave mixing of Ref. 22 which utilized an rf current pump $[11,13]$ in the vicinity of the signal frequency. We report on good measured performance in both the non-degenerate and degenerate modes [35] of the JPA, warranting its later integration into the nanocalorimetry setup.

\section{Devices}

Amplifying the readout signals of a nano-calorimeter requires sufficiently high dynamic range and enough bandwidth from the JPA. More specifically, the amplifier should be able to handle input signals at a maximum power of $-120 \mathrm{dBm}$ without going into saturation, and it needs to respond to detector signals in the time scales of $10-1000 \mu \mathrm{s}$. These targets were met in Ref. 22 using a JPA realized with a lumpedelement LC resonator for radio frequencies. The inductance originated largely from Josephson junctions in a series array of 200 SQUIDs with a maximal critical current of $35 \mu \mathrm{A}$. Shunted with a capacitance of $\simeq 30 \mathrm{pF}$, the flux-tunable resonator had a maximum resonance frequency $f_{0}$ of $650 \mathrm{MHz}$. The capacitive coupling to an external $50-\Omega$ rf environment set the gain-bandwidth product to $2 \pi f_{0} / Q_{e} \simeq 2 \pi \times 2.2 \mathrm{MHz}$, where $Q_{e} \simeq 300$ is the external quality factor. In this work, the device parameters are similar, and a detailed listing is provided in the Supplement [36].

The devices [Fig. 1(a)] incorporate an on-chip flux bias line (FBL) on a dedicated superconductive layer. Among many solutions for FBLs [12, 13, 37-40], our implementation has the advantage that the dc bias can be routed as a twisted pair through the cryostat 
while the rf pump tone propagates along the same onchip conductor. A simplified wiring schematic for the devices is shown in Fig. 1(b) and details are presented in the Supplement.

The devices are fabricated with an improved process that has been reported in detail in Ref. 32. In short, a high-resistivity silicon wafer is first cleaned of thermal oxides. Following that, the Josephson junctions are implemented with a niobium tri-layer $\left(\mathrm{Nb} / \mathrm{Al}^{-} \mathrm{Al}_{2} \mathrm{O}_{3} / \mathrm{Nb}\right)$ with thicknesses $100 \mathrm{~nm} / 10 \mathrm{~nm} / 100 \mathrm{~nm}$. The tri-layer is etched to a strip geometry and following the SWAPS process the sidewalls are passivated with PECVD silicon dioxide, as shown in a scanning electron micrograph in the Supplement. Crucially, the passivation step leaves no residual dielectric layer to the device area outside the junctions. The next step is the deposition of $120 \mathrm{~nm}$ of niobium for the main wiring layer. The Josephson junctions form wherever this layer crosses the tri-layer strips. Because of the need for the onchip FBL, we add a thin, 40-nm insulating layer of ALD $\mathrm{Al}_{2} \mathrm{O}_{3}$ with wet-etched contact holes. Finally, the FBL and some superconducting cross-overs are defined from $120 \mathrm{~nm}$ of niobium deposited as the topmost layer. The capacitors of the previous JPA design [22] were formed from parallel plates separated by a silicon dioxide layer, but here we use interdigitated fingers where the participation ratio of the lossy dielectrics is dramatically lower. The initial maximum resonance frequency of the devices at zero applied magnetic flux is about $500 \mathrm{MHz}$. We fine-tune it to a higher value by removing a part of the shunt capacitance with a focused ion beam (FIB) [Fig. 1(c)].

Two nominally identical devices $\mathrm{A}$ and $\mathrm{B}$ were prepared with the maximal resonance frequency targeted on $650 \mathrm{MHz}$, in order to make flux pumping feasible at the operating frequency of $600 \mathrm{MHz}$. The chip containing the JPA is placed on a holder and inside an aluminum-Amumetal 4K magnetic shield both of which are thermalized to the mixing stage of a dry dilution refrigerator. Prior to pumping, an initial characterization of the device takes place. It comprises the study of the small-signal response with a vector network analyzer (VNA) while tuning the resonance frequency with a magnetic flux, generated by a dc current applied to the FBL. A fit to the recorded reflection coefficient of the JPA allows us to determine the resonance frequency. Device A shows excellent reproducible tuneability between $520 \mathrm{MHz}$ and $667 \mathrm{MHz}$ [Fig. 1(d)] from which can be concluded that the SQUID array has a relatively homogeneous magnetic flux bias. Only a slight hysteresis occurs below $640 \mathrm{MHz}$ probably in part because of the geometric inductances [41]. In contrast, the previous generation amplifier had very irregular frequency response and only two viable operating points [22]. There are several factors that could play a role in the observed improvement. First, a ground plane with flux-trapping holes [42] has been added to the device layout. Second, we have increased the size of the SQUID loops by $65 \%$ to $2.1 \times 4.3 \mu \mathrm{m}^{2}$ so that less dc current is needed in the FBL. Finally, we have paid special attention to using non-magnetic materials in close proximity to the JPA chip.

\section{Gain and noise in the non-degenerate mode}

The JPA operating points defined by the triplet of the dc bias current, the associated flux pump frequency, and pump power are optimized and characterized by an automated procedure described in the Supplement. To study the gain and signal-to-noise ratio (SNR), we apply a weak probe tone at an offset of $-10 \mathrm{kHz}$ from the halved pump frequency where the JPA gain is maximal. The probe power is set to $-146 \mathrm{dBm}$ $(-136 \mathrm{dBm})$ at frequencies below (above) $580 \mathrm{MHz}$, to adjust accordingly to the dynamic range. In the data of Fig. 2(a), the SNR is optimized at each static-flux operating point, while constraining the maximum gain to $20.5 \mathrm{~dB}$. A gain of $18.5-20.5 \mathrm{~dB}$ is attained and the SNR improves by $15-18 \mathrm{~dB}$, as compared to the unity-gain reference where the noise floor is set by the HEMT post-amplifier. The independently measured noise added by the HEMT is $10-13 \mathrm{~K}$. The saturation of the JPA is investigated at the discovered operating points by varying the probe power [Fig. 2(b)] and it is found that the lower limit of $-120 \mathrm{dBm}$, required for calorimeter readout, is easily surpassed by about $10 \mathrm{~dB}$ at $600 \mathrm{MHz}$. After increasing the pump frequency slightly to lower the gain to $15 \mathrm{~dB}$, a gain-bandwidth product of $2 \pi \times 3 \mathrm{MHz}$ is measured [Fig. 2(c)]. It is sufficient for the detection of the thermal transients of the nano-calorimeter.

We investigate the potential range of the operating temperatures of Device A by setting a gain of $20 \mathrm{~dB}$ and measuring the JPA response with the VNA while heating the system. The frequency and power of the flux pump are kept at the fixed values. We attribute changes in the gain to the temperature dependence of the JPA resonance frequency. Raising the temperature from $40 \mathrm{mK}$ to $80 \mathrm{mK}(400 \mathrm{mK})$ increases the gain by $1 \mathrm{~dB}(5 \mathrm{~dB})$. The gain stays above $20 \mathrm{~dB}$ below $1.0 \mathrm{~K}$, and even at $1.5 \mathrm{~K}$ there is still a significant $16 \mathrm{~dB}$ of gain. The equivalent shift of the JPA resonance is $\pm 200 \mathrm{kHz}$, or $\pm 0.04 \%$. We note that these observations result from an interplay of several temperature-dependent quantities such as the permittivity of the $\mathrm{Al}_{2} \mathrm{O}_{3}$ coating of the JPA, the junction critical current, and the kinetic inductance of niobium [43]. 


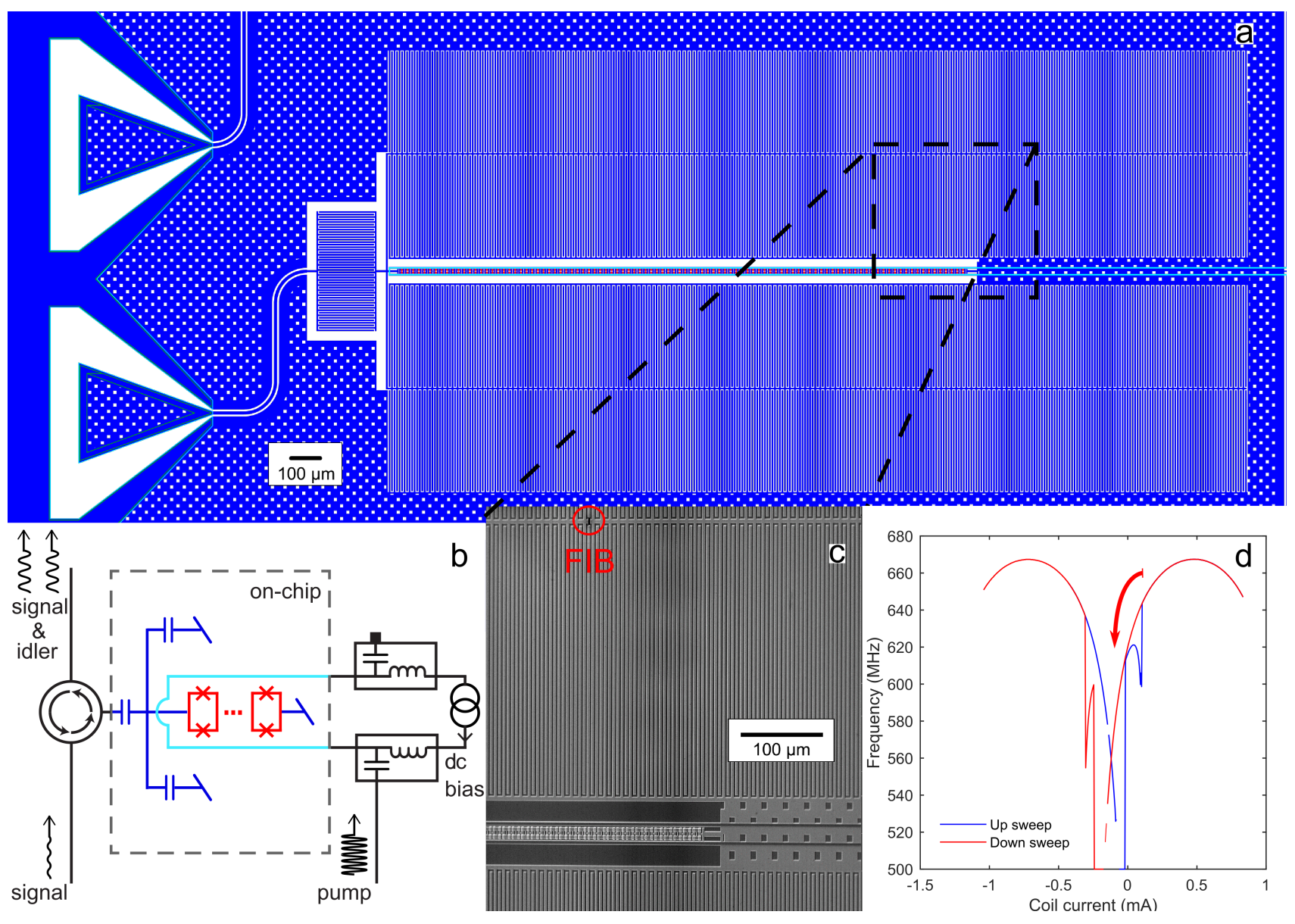

Figure 1. Layout and measurement of JPA. (a) Mask drawing showing the whole JPA with the SQUID array in red and an on-chip flux bias line (FBL) in light blue. The wiring layer in dark blue features bonding pads, shunt and coupling capacitors, and the ground plane. The holes in the ground plane trap magnetic flux. (b) Simplified measurement setup with the JPA enclosed in the dashed box. The FBL carries both a pump tone and a dc current used to bias the SQUIDs and, thus, modify the resonance frequency of the device. The probe tone enters through a circulator and three-wave mixing within the JPA allows the pump tone to produce signal and idler photons according to $f_{\text {pump }}=f_{\text {probe }}+f_{\text {idler }}$. A second circulator (not shown) further enhances the isolation from a subsequent cascode of post-amplifiers. (c) Scanning electron micrograph showing a part of the SQUID array and the interdigital shunt capacitor cut with a focused ion beam to fine-tune the resonance. A close-up of the other end of the array is shown in the Supplement. (d) Tuneability of the resonance frequency of Device A. Shown are a single up sweep and a down sweep of current measured with the pump off. The arrow indicates a deterministic branch selected for subsequent measurements.

To better estimate the noise added by the JPA, the system noise temperature is determined with the Y-factor method [44]. Essentially, the power spectral density at the output of the JPA is surveyed with a spectrum analyzer while the JPA is subject to an impedance-matched resistive noise source with a controlled temperature. The source temperature is varied between $59 \mathrm{mK}$ and $852 \mathrm{mK}$ independently of the JPA temperature that is held constant at $30 \mathrm{mK}$ with closed-loop control. The measurements are carried out with the Device B and the full setup is shown in the Supplement. The system noise temperature, referenced to the JPA input, takes its minimum value of $165 \mathrm{mK}$ close to the halved pump frequency [Fig. 4(a)]. Since the noise added by the JPA is reasonably independent of the offset
[Fig. 4(b)], we may take the average with the inverted variances as weights. This yields a noise estimate of $105 \mathrm{mK} \pm 9 \mathrm{mK}$. Making the comparison to Ref. 22, we note that the added noise has approximately halved. Also notable is that the noise is no longer at an elevated level at frequencies close to the gain maximum, which may be because the rf pump tone has been moved away from it to the double frequency.

\section{Degenerate gain}

In a dispersive sensor, such as the nano-calorimeters of Ref. 24, it is possible to choose the excitation and readout in such a way that the rf carrier and the information-carrying signal are in different quadratures. In this situation, it is possible to utilize 


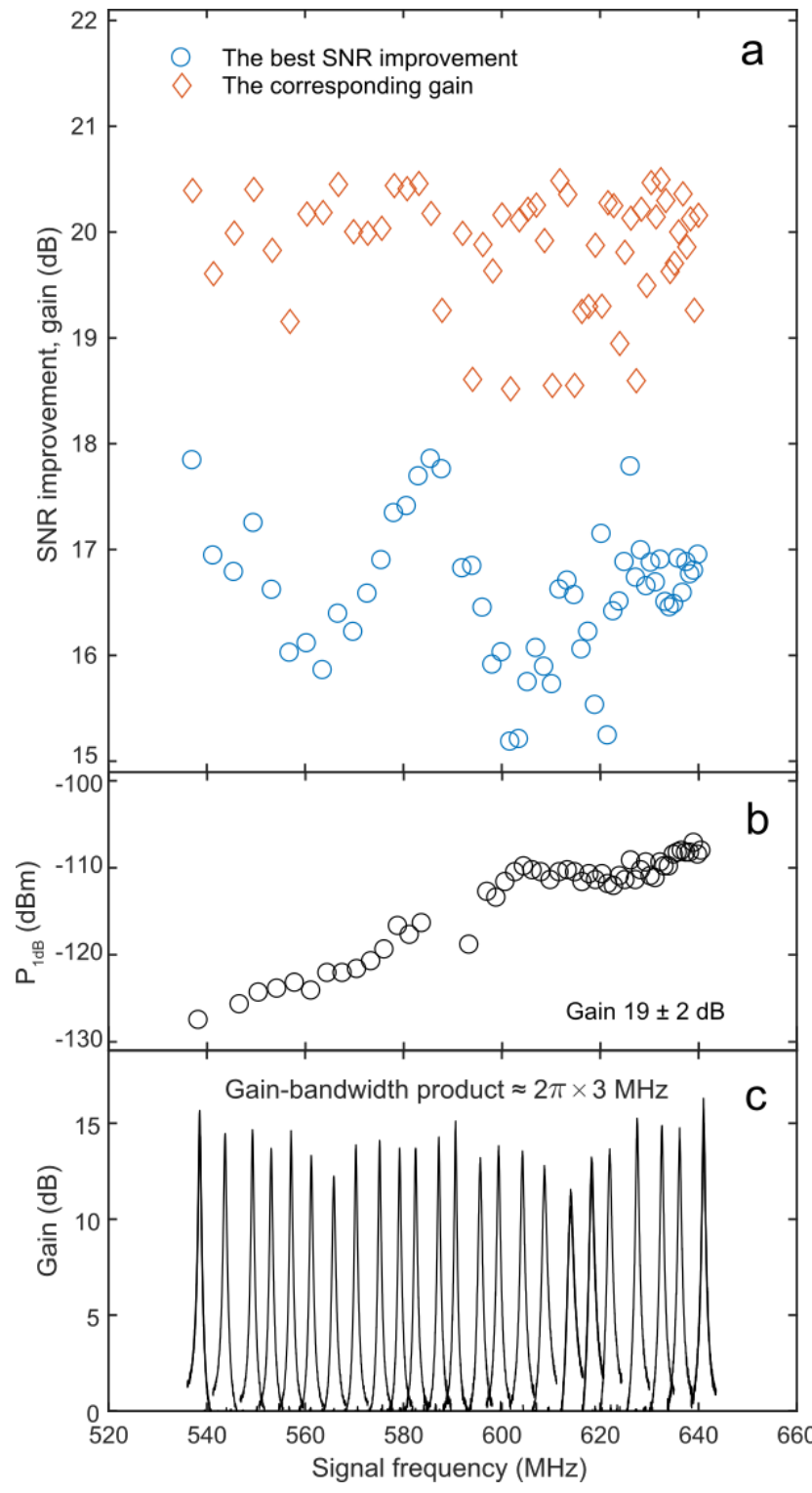

Figure 2. Systematic characterization of Device A at $10 \mathrm{mK}$. At each operating point determined by a static flux bias current, the pump power is optimized first and the gain is then adjusted with the pump frequency. (a) The best signal-to-noise ratio improvement and corresponding gain while limiting to gains under $20.5 \mathrm{~dB}$. The quantities are in respect to a reference that is measured with the JPA detuned and the pump switched off. (b) 1-dB compression points of gain at a probe offset of $-10 \mathrm{kHz}$ from the halved pump frequency where the gain is maximal. At around $590 \mathrm{MHz}$ the pumping was unsuccessful. (c) Lorentzian gain profiles as a function of the signal frequency at 24 selected operating points. The target gain of each measurement was $15 \mathrm{~dB}$.

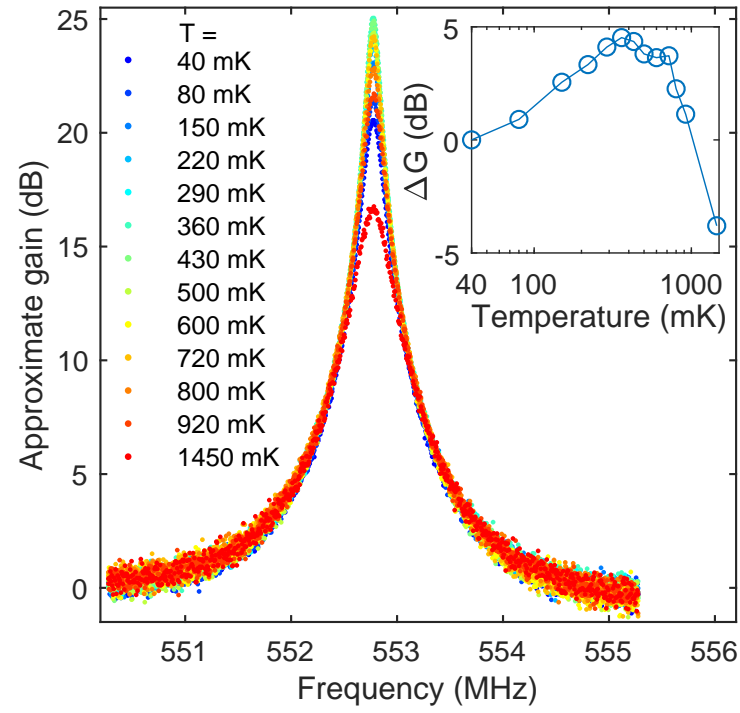

Figure 3. Approximate gain profiles for Device A at $553 \mathrm{MHz}$ at varying temperatures of the sample stage starting from a gain of $20 \mathrm{~dB}$ at $40 \mathrm{mK}$. In the absence of reference data, the recorded signal is normalized to the edges of the peak. The pump power or frequency are not re-optimized during heating proving the temperature robustness of the amplifier. Inset: change in gain from $40 \mathrm{mK}$, as a function of temperature.

squeezing to de-amplify the carrier and to amplify the signal. This may be used to effectively increase the dynamic range of the later stages of the readout. Ideally, the amplification does not add any noise if a JPA is used to perform the squeezing.

To observe squeezing in the degenerate mode of the JPA, where the pump is exactly at twice the probe frequency, a brief experiment is carried out with the Device B. Using a single rf source, the pump is synthesized with a frequency doubler and the relative phase $\theta$ of the probe is controlled as shown in Fig. 5(a). The JPA gain as a function of $\theta$ is $\pi$-periodic as expected, and amplification and de-amplification alternate. The JPA is thus capable of squeezing [45]. However, here we do not attempt a proper investigation of the quadratures to determine how much vacuum squeezing is attainable. 


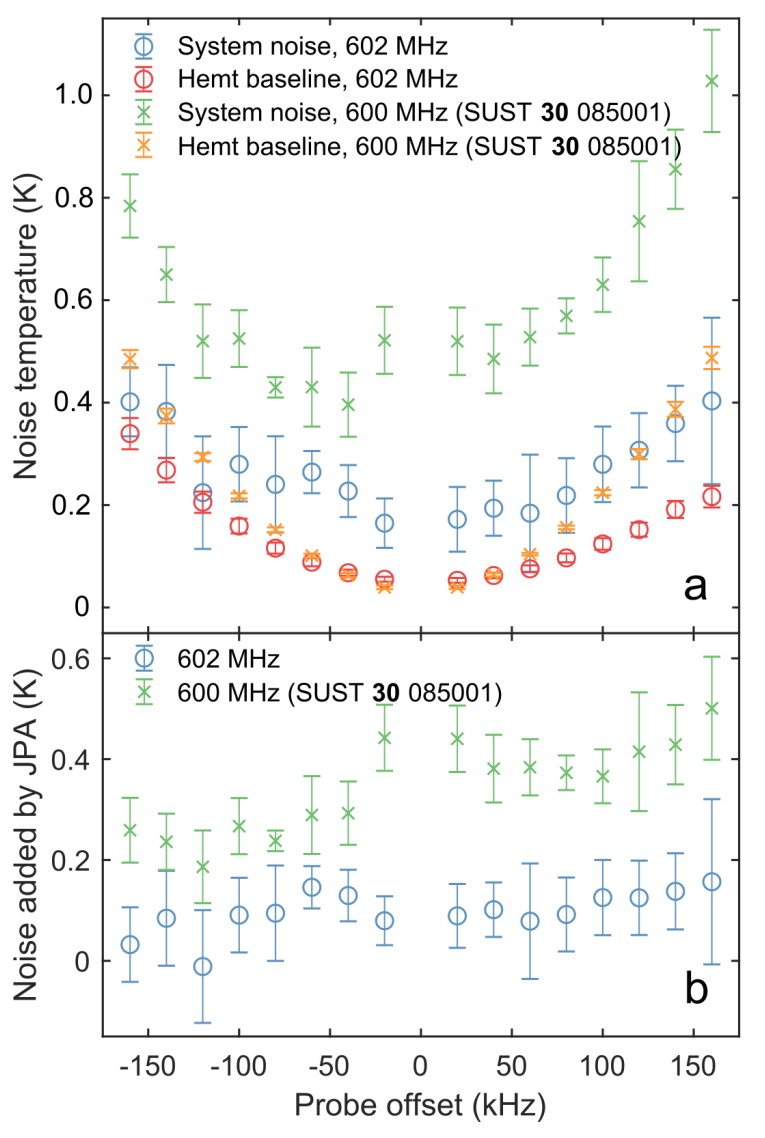

Figure 4. Noise temperature of Device B at $600 \mathrm{MHz}$ determined with the $\mathrm{Y}$-factor method using a calibrated noise source. Data from Ref. 22 are added for comparison. (a) System noise temperature $T_{\text {sys }}$ as a function of the probe offset from the halved pump frequency. The separated contribution of the HEMT amplifier to $T_{\text {sys }}$ is the smallest at zero offset where the JPA gain is maximal. (b) The noise added by the JPA is the difference between the system noise temperature and the part added by HEMT as well as the input noise: $30 \mathrm{mK}$ in recent experiments and $40 \mathrm{mK}$ in Ref. 22 .

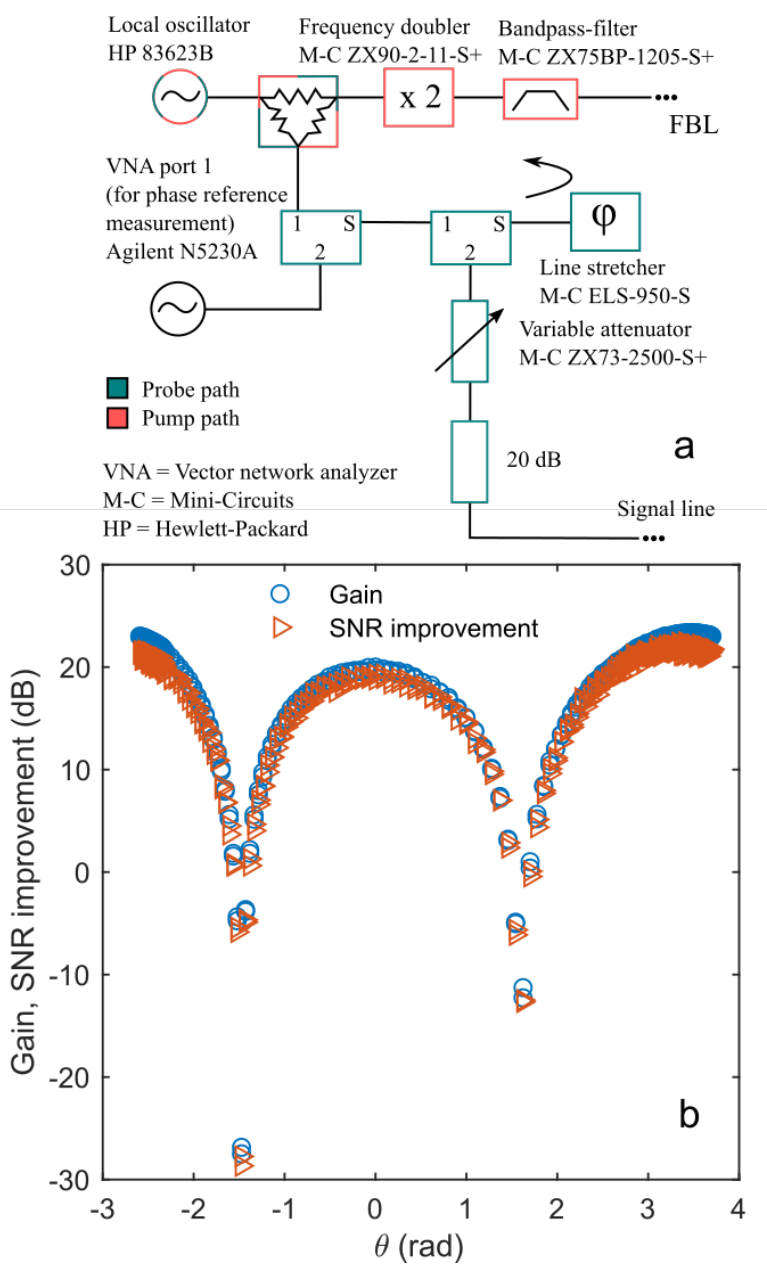

Figure 5. Device $B$ in the degenerate mode at an operating temperature of $30 \mathrm{mK}$. The probe frequency reads $f_{\text {probe }}=$ $\frac{1}{2} f_{\text {pump }}=f_{\text {idler }}=601.85 \mathrm{MHz}$. (a) A frequency doubler generates the phase-locked pump tone. The probe is directed to a voltage-controlled electronic line stretcher (ELS) to adjust the phase difference between the probe and the pump. Afterwards, the VNA is used to calibrate the phase versus the control voltage. (b) Gain (circles) and SNR improvement (triangles) as a function of the calibrated phase. The latter is determined by comparing to a measurement where the JPA is detuned with applied magnetic flux. The observed difference of $3 \mathrm{~dB}$ between the gain maxima is likely caused by varying pump power, due to voltage dependence in the reflection coefficient of the ELS: a signal leaking backwards to the input of the doubler interferes with the signal following the direct path. 


\section{Conclusion}

We have overcome all the major issues discovered with the previous-generation JPA [22], and we have attained robust performance over a wide frequency range without sacrificing bandwidth, gain, or dynamic range. Additionally, we have minimized the amount of TLS-hosting dielectrics and their participation ratio with the SWAPS [32] fabrication process. This renders the JPA relatively insensitive to temperature, facilitating its use at variable mK-temperatures as only minor corrections to the JPA operating parameters are needed. Furthermore, the JPA is now flux-pumped at twice the readout frequency of the nano-calorimeter, easing the filtering required to avoid back-action such as residual heating by the pump. As an important step towards sub-GHz sensor readout at a fidelity beyond the standard quantum limit [46], squeezing has been observed in the degenerate mode of the JPA.

The noise added by the JPA has decreased to $105 \mathrm{mK}$ at $600 \mathrm{MHz}$. This can largely be attributed to better isolation from the HEMT post-amplifier by means of an additional circulator. However, the added noise remains at an elevated level with respect to the lower bound set by the input thermal noise at $30 \mathrm{mK}$, likely due to poorly thermalized attenuators [47] or a fundamental limit of the amplifier itself. The noise performance also remains inferior to that of a microstrip SQUID amplifier with a reported noise temperature of $48 \mathrm{mK}$ at $612 \mathrm{MHz}$ [44]. Yet, we have demonstrated a sub-GHz JPA that is well suited for integration into a nano-calorimetry [24] or -bolometry [26] apparatus and we will pursue the latter goal in a future experiment.

\section{Acknowledgments}

We thank Paula Holmlund for sample preparation, Harri Pohjonen for help with lithographic masks, and Mikko Kiviranta for operating the FIB. We acknowledge the fruitful discussions with Olli-Pentti Saira, Roope Kokkoniemi, Mikko Möttönen, and Jukka Pekola. This work was performed as part of the Academy of Finland Centre of Excellence program (projects 284594, 312059, 251748, and 284621). The work also received funding from Academy of Finland project QuMOS (project numbers 288907 and 287768), Future Makers Funding Program by Technology Industries of Finland Centennial Foundation and Jane and Aatos Erkko Foundation. 


\section{References}

[1] Bradley R, Clarke J, Kinion D, Rosenberg L J, van Bibber K, Matsuki S, Mück M and Sikivie P 2003 Reviews of Modern Physics 75777

[2] Asztalos S J, Carosi G, Hagmann C, Kinion D, van Bibber K, Hotz M, Rosenberg L J, Rybka G, Hoskins J, Hwang J, Sikivie P, Tanner D B, Bradley R and Clarke J 2010 Physical Review Letters 104041301

[3] Kenany S A, Anil M A, Backes K M, Brubaker B M, Cahn S B, Carosi G, Gurevich Y V, Kindel W F, Lamoreaux S K, Lehnert K W, Lewis S M, Malnou M, Palken D A, Rapidis N M, Root J R, Simanovskaia M, Shokair T M, Urdinaran I, van Bibber K A and Zhong L 2017 Nuclear Instruments and Methods in Physics Research Section A: Accelerators, Spectrometers, Detectors and Associated Equipment 854 11-24

[4] Bergeal N, Schackert F, Metcalfe M, Vijay R, Manucharyan V E, Frunzio L, Prober D E, Schoelkopf R J, Girvin S M and Devoret M H 2010 Nature 465 64-68

[5] Abdo B, Sliwa K, Shankar S, Hatridge M, Frunzio L, Schoelkopf R and Devoret M 2014 Physical Review Letters 112167701

[6] O'Brien K, Macklin C, Hover D, Schwartz M E, Bolkhovsky V, Zhang X, Oliver W D and Siddiqi I 2016 Towards quantum-noise limited multiplexed microwave readout of qubits 2016 IEEE MTT-S International Microwave Symposium (IMS) pp 13

[7] Devoret M H and Schoelkopf R J 2013 Science 339 1169-1174

[8] Calusine G, Melville A, Woods W, Das R, Stull C, Bolkhovsky V, Braje D, Hover D, Kim D K, Miloshi X, Rosenberg D, Sevi A, Yoder J L, Dauler E and Oliver W D 2018 Applied Physics Letters 112062601

[9] Clark J B, Lecocq F, Simmonds R W, Aumentado J and Teufel J D 2016 Nature Physics 12 683-687

[10] Yurke B, Kaminsky P G, Miller R E, Whittaker E A, Smith A D, Silver A H and Simon R W 1988 Physical Review Letters 60 764-767

[11] Castellanos-Beltran M A and Lehnert K W 2007 Applied Physics Letters 91083509

[12] Yamamoto T, Inomata K, Watanabe M, Matsuba K, Miyazaki T, Oliver W D, Nakamura Y and Tsai J S 2008 Applied Physics Letters 93042510

[13] Mutus J Y, White T C, Jeffrey E, Sank D, Barends R, Bochmann J, Chen Y, Chen Z, Chiaro B, Dunsworth A, Kelly J, Megrant A, Neill C, O'Malley P J J, Roushan P, Vainsencher A,
Wenner J, Siddiqi I, Vijay R, Cleland A N and Martinis J M 2013 Applied Physics Letters 103 122602

[14] Eichler C and Wallraff A 2014 EPJ Quantum Technology 12

[15] Mutus J Y, White T C, Barends R, Chen Y, Chen Z, Chiaro B, Dunsworth A, Jeffrey E, Kelly J, Megrant A, Neill C, O'Malley P J J, Roushan P, Sank D, Vainsencher A, Wenner J, Sundqvist K M, Cleland A N and Martinis J M 2014 Applied Physics Letters 104263513

[16] Eichler C, Salathe Y, Mlynek J, Schmidt S and Wallraff A 2014 Physical Review Letters 113 110502

[17] Vissers M R, Erickson R P, Ku H S, Vale L, Wu X, Hilton G C and Pappas D P 2016 Applied Physics Letters 108012601

[18] Mallet F, Castellanos-Beltran M A, Ku H S, Glancy S, Knill E, Irwin K D, Hilton G C, Vale L R and Lehnert K W 2011 Physical Review Letters 106220502

[19] Fedorov K G, Zhong L, Pogorzalek S, Eder P, Fischer M, Goetz J, Xie E, Wulschner F, Inomata $\mathrm{K}$, Yamamoto $\mathrm{T}$, Nakamura $\mathrm{Y}$, Di Candia R, Las Heras U, Sanz M, Solano E, Menzel E P, Deppe F, Marx A and Gross R 2016 Physical Review Letters 117020502

[20] Bienfait A, Campagne-Ibarcq P, Kiilerich A, Zhou X, Probst S, Pla J, Schenkel T, Vion D, Esteve D, Morton J, Moelmer K and Bertet P 2017 Physical Review X 7041011

[21] Clerk A A, Devoret M H, Girvin S M, Marquardt F and Schoelkopf R J 2010 Reviews of Modern Physics 82 1155-1208

[22] Vesterinen V, Saira O P, Räisänen I, Möttönen M, Grönberg L, Pekola J and Juha Hassel 2017 Superconductor Science and Technology 30 085001

[23] Gasparinetti S, Viisanen K L, Saira O P, Faivre T, Arzeo M, Meschke M and Pekola J P 2015 Physical Review Applied 3014007

[24] Viisanen K L, Suomela S, Gasparinetti S, Saira O P, Ankerhold J and Pekola J P 2015 New Journal of Physics 17055014

[25] Govenius J, Lake R E, Tan K Y, Pietilä V, Julin J K, Maasilta I J, Virtanen P and Möttönen M 2014 Physical Review B 90064505

[26] Govenius J, Lake R E, Tan K Y and Möttönen M 2016 Physical Review Letters 117030802

[27] Karasik B S and Cantor R 2011 Applied Physics Letters $\mathbf{9 8} 193503$ 
[28] Suzuki T, Khosropanah P, Hijmering R A, Ridder M, Schoemans M, Hoevers $\mathrm{H}$ and Gao J R 2014 IEEE Transactions on Terahertz Science and Technology 4 171-178

[29] Penfold-Fitch Z V, Sfigakis F and Buitelaar M R 2017 Physical Review Applied 7054017

[30] O'Connell A D, Ansmann M, Bialczak R C, Hofheinz M, Katz N, Lucero E, McKenney C, Neeley M, Wang H, Weig E M, Cleland A N and Martinis J M 2008 Applied Physics Letters 92 112903

[31] Pappas D P, Vissers M R, Wisbey D S, Kline J S and Gao J 2011 IEEE Transactions on Applied Superconductivity 21 871-874

[32] Grönberg L, Kiviranta M, Vesterinen V, Lehtinen J, Simbierowicz S, Luomahaara J, Prunnila M and Hassel J 2017 Superconductor Science and Technology 30125016

[33] Kiviranta M, Brandel O, Grönberg L, Kunert J, Linzen S, Beev N, May T and Prunnila M 2016 IEEE Transactions on Applied Superconductivity $261-5$

[34] Lähteenmäki P, Paraoanu G S, Hassel J and Hakonen P J 2013 Proceedings of the National Academy of Sciences 110 4234-4238

[35] Devoret M and Roy A 2016 Comptes Rendus Physique 17 740-755

[36] Supplementary information URL Supplementary information available online at.

[37] Sandberg M, Wilson C M, Persson F, Bauch T, Johansson G, Shumeiko V, Duty T and Delsing P 2008 Applied Physics Letters 92203501

[38] Krantz P, Reshitnyk Y, Wustmann W, Bylander J, Gustavsson S, Oliver W D, Duty T, Shumeiko V and Delsing P 2013 New Journal of Physics 15 105002

[39] Svensson I M, Bengtsson A, Krantz P, Bylander J, Shumeiko V and Delsing P 2017 Physical Review B 96174503

[40] Zhou X, Schmitt V, Bertet P, Vion D, Wustmann W, Shumeiko V and Esteve D 2014 Physical Review B 89214517

[41] Pogorzalek S, Fedorov K G, Zhong L, Goetz J, Wulschner F, Fischer M, Eder P, Xie E, Inomata K, Yamamoto T, Nakamura Y, Marx A, Deppe F and Gross R 2017 Physical Review Applied 8 024012

[42] Chiaro B, Megrant A, Dunsworth A, Chen Z, Barends R, Campbell B, Chen Y, Fowler A, Hoi I C, Jeffrey E, Kelly J, Mutus J, Neill C, O'Malley P J J, Quintana C, Roushan P, Sank D, Vainsencher A, Wenner J, White T C and Martinis
J M 2016 Superconductor Science and Technology 29104006

[43] Annunziata A J, Santavicca D F, Frunzio L, Catelani G, Rooks M J, Frydman A and Prober D E 2010 Nanotechnology 21445202

[44] Kinion D and Clarke J 2011 Applied Physics Letters $\mathbf{9 8} 202503$

[45] Zhong L, Menzel E P, Di Candia R, Eder P, Ihmig M, Baust A, Haeberlein M, Hoffmann E, Inomata K, Yamamoto T, Nakamura Y, Solano E, Deppe F, Marx A and Gross R 2013 New Journal of Physics 15125013

[46] Caves C M 1982 Physical Review D 26 1817-1839

[47] Yeh J H, LeFebvre J, Premaratne S, Wellstood F C and Palmer B S 2017 Journal of Applied Physics 121224501 


\title{
Supplement for article "Flux-driven Josephson parametric amplifier for sub-GHz frequencies fabricated with side-wall passivated spacer junction technology"
}

\author{
Slawomir Simbierowicz ${ }^{1}$, Visa Vesterinen ${ }^{1,2}$, Leif Grönberg ${ }^{1}$, \\ Janne Lehtinen ${ }^{1}$, Mika Prunnila ${ }^{1}$ and Juha Hassel ${ }^{1}$ \\ ${ }^{1}$ VTT Technical Research Centre of Finland Ltd \& QTF Centre of Excellence, \\ P.O.Box 1000, FI-02044 VTT, FINLAND \\ ${ }^{2}$ QCD Labs, COMP Centre of Excellence, Department of Applied Physics, Aalto \\ University, P.O. Box 13500, 00076 Aalto, Finland \\ E-mail: slawomir.simbierowicz@vtt.fi
}

May 2018 


\section{Dynamic range}

The limitation of dynamical range at low frequency is circumvented by adding 200 SQUIDs in series, enabled by the expression for the bifurcation power of a parametric current pump [1]:

$$
P_{\mathrm{bf}}=\frac{N^{2} \chi^{2} Q \phi_{0}^{2} \omega_{0}^{2}}{32 \sqrt{3} \eta Q_{\mathrm{e}} Z_{\mathrm{LC}}},
$$

where $N$ is the number of Josephson elements in series, $\omega_{0} /(2 \pi)$ is the drive frequency of the device, $Q$ and $Q_{\mathrm{e}}$ are the total and external quality factors, and $\chi=\frac{16}{\sqrt{3}} Q^{-1} \eta^{-1}$. The quantity $\eta=1 /\left(1+L_{\text {geom }}^{(1)} / L_{\mathrm{J}}^{(1)}\right)$ is controlled with the geometric and Josephson inductances. Finally, $\phi_{0}=\hbar /(2 e)$ is the reduced flux quantum. Our devices were designed using a ratio of $L_{\text {geom }}^{(1)} / L_{\mathrm{J}}^{(1)}=0.30$ and a characteristic impedance of $Z_{\mathrm{LC}}=$ $\sqrt{L / C}=8.8 \Omega$, where $L=L_{\text {geom }}^{(1)}+L_{\mathrm{J}}^{(1)}$ and $C$ is the total capacitance of the resonator. Determining quality factors from experiments performed on Device A, we get $Q_{\mathrm{e}}=230$ and $Q_{\mathrm{i}}=1771$ at $601 \mathrm{MHz}$. Then, $\chi=0.06$ and $P_{\mathrm{bf}}=-92.9 \mathrm{dBm}$ which is $1 \mathrm{~dB}$ higher than in Ref. 1 and sufficient for amplifying nano-calorimeter signals.

\section{Junction structure}

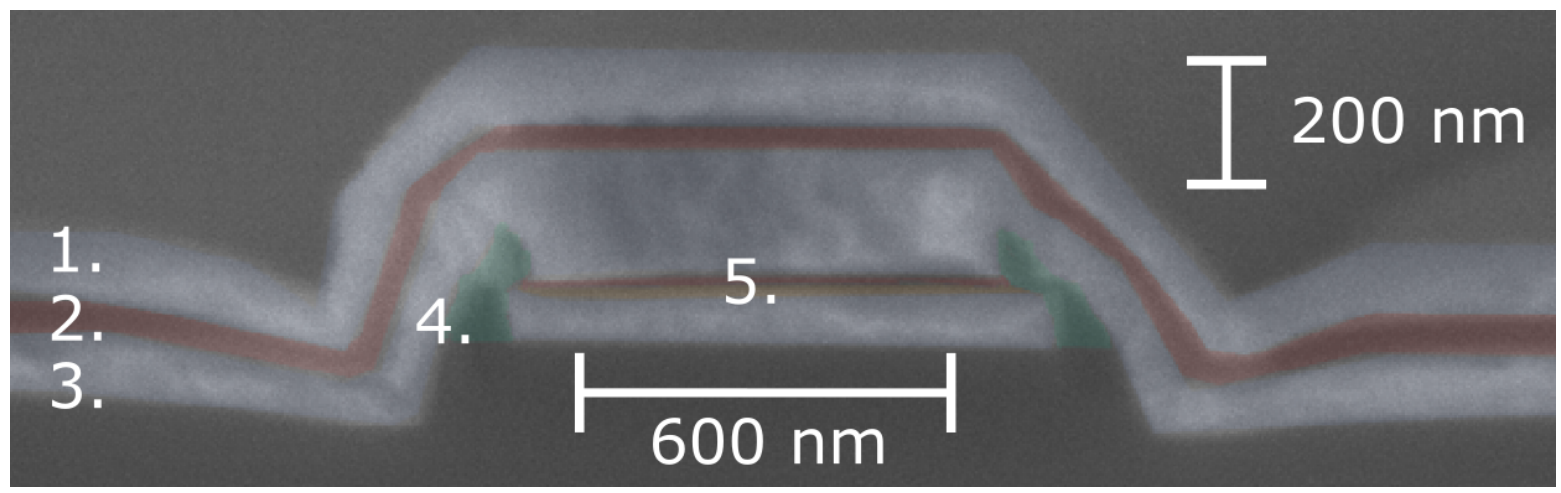

Figure 1. False colored scanning electron micrograph showing a cross-section of a test junction sharing the layer composition of the JPAs. A high-resistivity silicon substrate carries the structure with the following elements: 1 . the niobium cross-overs and the flux bias line; 2. the insulating ALD aluminium oxide layer; 3 . the niobium ground plane and wiring layer; 4 . a silicon dioxide spacer used for the passivation of junction side-walls; 5 . the $\mathrm{Nb}-\mathrm{Al} / \mathrm{AlO}_{\mathrm{x}}-\mathrm{Nb}$ trilayer containing the tunnel junctions. 
SQUID close-up

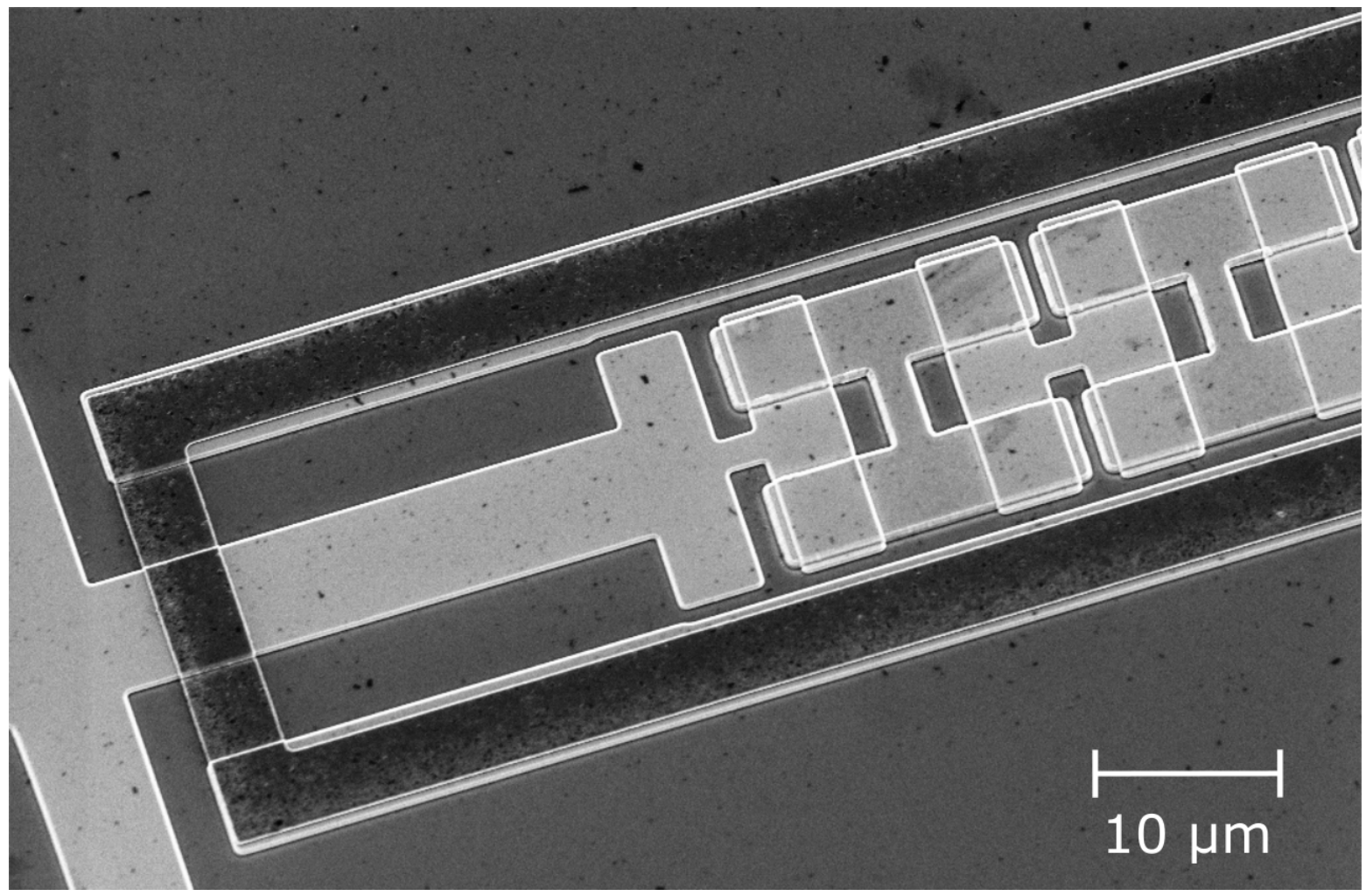

Figure 2. Scanning electron micrograph showing one end of the niobium SQUID array. The flux bias line (black) can be seen crossing over the center conductor. 


\section{Full cryogenic setup}

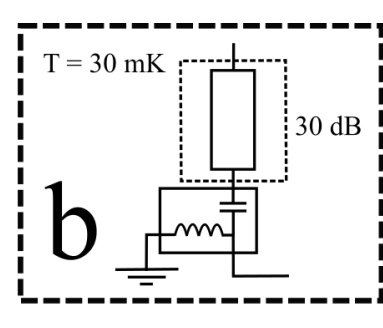

Probe (VNA port 1) Agilent N5230A

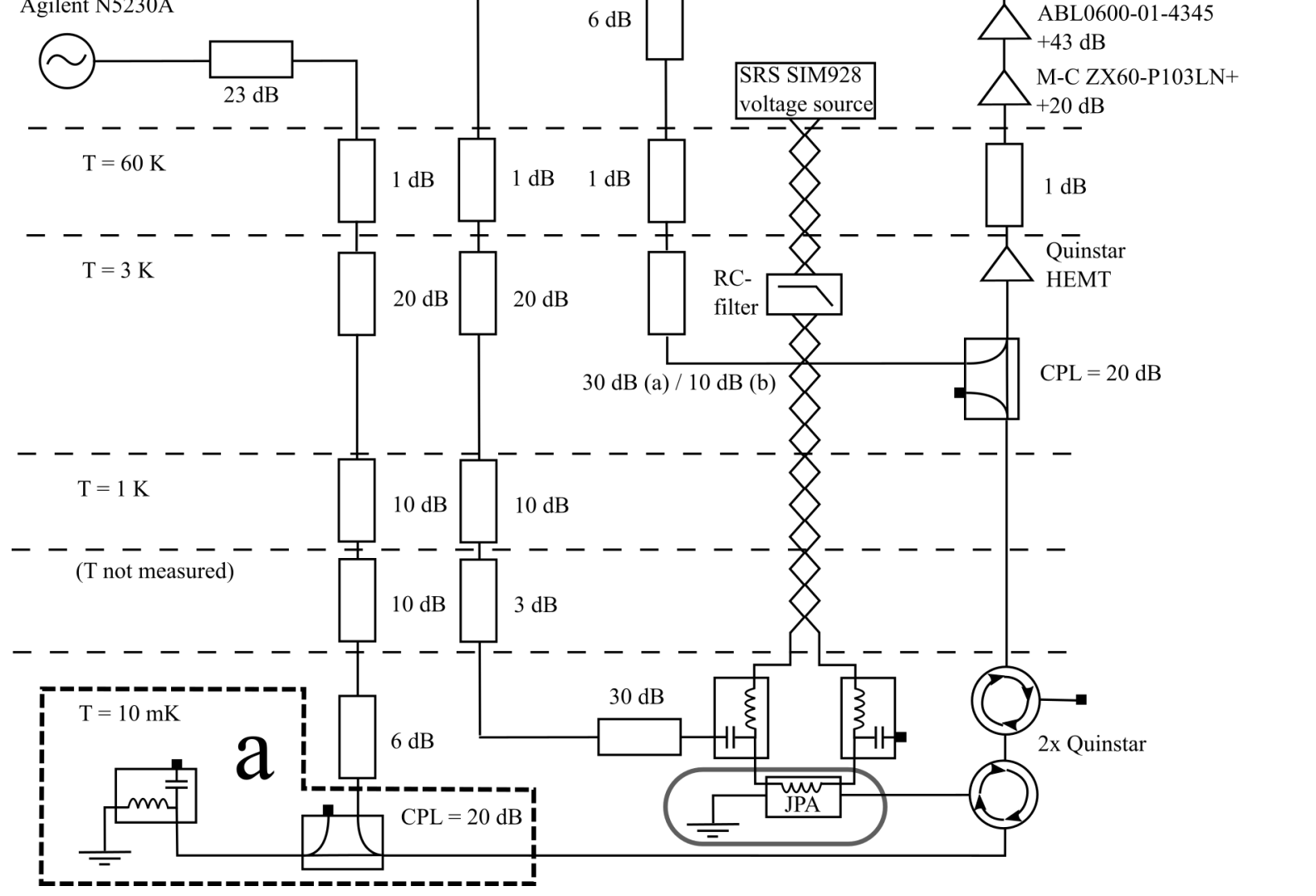

Figure 3. Detailed wiring schematic. The JPA lies inside an aluminum-Amumetal $4 \mathrm{~K}$ magnetic shield (gray line) thermalized to the mixing plate (MP) of a dilution refrigerator (BlueFors Cryogenics LD-250). A twisted pair of wires carries DC current to the on-chip coil biasing the SQUID chain on the device. The DC bias is combined with the RF pump using two bias-tees: the second one terminates the pump line. Both probe and pump RF tones are heavily attenuated in steps. Two back-to-back circulators protect the JPA from HEMT $\left(T_{\mathrm{N}}=10-13 \mathrm{~K}\right)$ back-action. Gain and noise measurements are performed using the VNA and the spectrum analyzer. The redundant RF line was previously used for cancellation of the pump tone during current pumping. All RF instruments are synchronized with a rubidium frequency standard (SRS FS725, not shown). (a) Measurement of Device A happens at $10 \mathrm{mK}$. The combination of the directional coupler and the bias-tee thermalizes the JPA to the MP. (b) Measurements for Device B are performed at $30 \mathrm{mK}$ and the signal is rerouted through a noise source: a heated $30 \mathrm{~dB}$ attenuator (XMA Corp.) inside a copper enclosure (small dashed box) weakly thermalized to the MP. 


\section{Automated pump procedure}

Before executing the automated pumping procedure, some parameters need to be selected: the range of dc coil currents, the probe power, the probe offset from the pump, and the power window during the probe power sweep that measures the 1-dB saturation point of the amplifier. Except in the saturation measurement, the probe power should be low enough that a small change in power does not affect the gain and high enough for an adequate signal-to-noise ratio, especially when the pump has been turned off. We use a spectrum analyzer to determine SNR and gain accurately. Additionally, in order to benefit from the higher dynamic range at high signal frequencies, the probe power is boosted by $10 \mathrm{~dB}$ there. Choosing the parameters may require manual operation of the amplifier.

The automated script is executed at each dc coil current. It starts by fitting to S-parameter data procured by the VNA and sets the pump frequency to $-3 \mathrm{MHz}$ from twice the obtained resonance frequency. For an expected gain-bandwidth product of $3 \mathrm{MHz}$, this puts the halved pump at a frequency offset reasonably detuned from the JPA resonance. Next, the pump power is increased until the JPA goes into the mode of parametric oscillation [2] which manifests itself as a strong peak measured with the spectrum analyzer at the halved pump frequency. The power is then lowered in steps of $0.1 \mathrm{dBm}$ until the oscillation disappears. We call this point the bifurcation power because of the hysteresis of the parametric oscillation. We further decrease the pump power by $0.15 \mathrm{dBm}$, arriving right below the so-called line of maximum gain [3]. After this, the pump frequency is increased in steps of $100 \mathrm{kHz}$ to find the range of available gain while the probe trails the halved pump frequency by the predefined offset of $-10 \mathrm{kHz}$. The spectrum analyzer is used to measure the probe power and the noise in a $20 \mathrm{kHz}$ window centered at the probe frequency.

Whenever reaching a gain threshold of $20 \mathrm{~dB}$, the user-defined sweep of the probe power is performed with the VNA. Similarly, at a gain threshold of $15 \mathrm{~dB}$ the VNA measures the gain-bandwidth product using a $5 \mathrm{MHz}$ window centered at the gain maximum. To calibrate the gain, the JPA is momentarily detuned and the pump is turned off to measure the reference without changing the settings of the VNA. Finally, the script re-visits all the frequency windows on the spectrum analyzer but with the JPA detuned and the pump turned off. This establishes the references for the probe power and the noise. 


\section{References}

[1] Vesterinen V, Saira O P, Räisänen I, Möttönen M, Grönberg L, Pekola J and Juha Hassel 2017 Superconductor Science and Technology 30085001

[2] Krantz P, Reshitnyk Y, Wustmann W, Bylander J, Gustavsson S, Oliver W D, Duty T, Shumeiko V and Delsing P 2013 New Journal of Physics 15105002

[3] Manucharyan V E, Boaknin E, Metcalfe M, Vijay R, Siddiqi I and Devoret M 2007 Physical Review B $\mathbf{7 6} 014524$ 